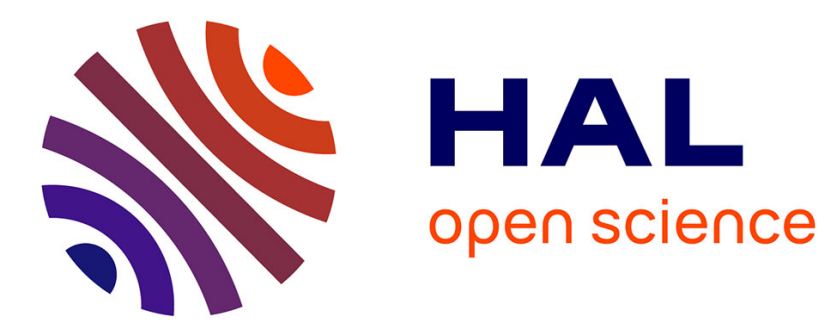

\title{
Nanotip response to monocycle terahertz pulses
}

\author{
J. Houard, L. Arnoldi, A. Ayoub, A. Hideur, A. Vella
}

\section{To cite this version:}

J. Houard, L. Arnoldi, A. Ayoub, A. Hideur, A. Vella. Nanotip response to monocycle terahertz pulses. Applied Physics Letters, 2020, 117 (15), pp.151105. 10.1063/5.0022182 . hal-02968928

\section{HAL Id: hal-02968928 \\ https://hal.science/hal-02968928}

Submitted on 16 Oct 2020

HAL is a multi-disciplinary open access archive for the deposit and dissemination of scientific research documents, whether they are published or not. The documents may come from teaching and research institutions in France or abroad, or from public or private research centers.
L'archive ouverte pluridisciplinaire HAL, est destinée au dépôt et à la diffusion de documents scientifiques de niveau recherche, publiés ou non, émanant des établissements d'enseignement et de recherche français ou étrangers, des laboratoires publics ou privés. 
Submitted to Appl. Phys. Lett.

\title{
Nanotip response to monocycle terahertz pulses
}

\author{
J. Houard, ${ }^{1}$ L. Arnoldi, ${ }^{1}$ A. Ayoub,${ }^{1}$ A.Hideur, ${ }^{2}$ and A. Vella ${ }^{1, *}$ \\ ${ }^{1}$ Groupe de Physique des Matériaux UMR CNRS 6634 - Université et INSA de ROUEN, \\ Université Normandie 76801 SAINT ETIENNE DU ROUVRAY CEDEX France \\ ${ }^{2}$ CORIA, UMR CNRS 6614 - Université et INSA de ROUEN, \\ Université Normandie 76801 SAINT ETIENNE DU ROUVRAY CEDEX France
}

(Dated: September 20, 2020)

\begin{abstract}
The interaction of monocycle terahertz ( $\mathrm{THz}$ ) fields with metal nanostructures such as nanootips is attracting a lot of interest for the development of new applications such as THz-scanning tunneling microscopy (STM) and ultra-fast field emission. New methods have been introduced to measure the enhanced $\mathrm{THz}$ near field waveform in the vicinity of a nanotip surface. Here we present results obtained by electron field emission in the multiphoton emission regime. The nanotip mimics the behavior of an antenna with a simplified equivalent circuit. We show that the electrical characteristics can be calculated using the nanowire geometry approximation and taking into account a conduction shell with a thickness smaller than the material skin depth. The results introduce a method to predict the antenna response of the nanotip and thus the near $\mathrm{THz}$ field waveform.
\end{abstract}


Terahertz radiations with low energetic photons $(\mathrm{meV})$ are used today in a wide range of applications such as imaging, sensing or spectroscopy. This low photon energy has the advantage to induce low damages on fragile materials, as biologic samples, or avoid strong heating encountered when visible wavelength is used[1]. Thanks to high intensity ultrafast lasers, different processes, such as photoconductive antenna [2,3], optical rectification in crystals[4, 5], or plasma generation in fluids[6,7], can be used to create pulsed sources of strong $\mathrm{THz}$ field $(\mathrm{kV} / \mathrm{cm})$. The intensity of these pulses can be enhanced through coupling with nanoscale object for different applications[8, 9]. For example, nanoscale tips can amplify the THz field for scanning application with temporal and spatial confinement in scattering-type scanning near-field optical microscopy (s-SNOM)[10], or for controlling/creating ultrashort electron pulses[11-13]. However, although THz electromagnetic waves are enhanced at the nanotip apex, the electric field waveform is also modulated simultaneously, as has been experimentally confirmed in SNOM and THz-STM[14, 15]. Although the theoretical description of the antenna effect has already been discussed[14, 16], precise measurements have proven to be very useful for directly measuring and quantitatively evaluating the waveform in actual measurement environments[17]. However, these measurements have been conducted only in the context of tunneling Thz-STM.

Here, we present the extension of this approach to measurements of the enhanced terahertz near field during multi-photon photoelectron emission in the femtosecond range. Moreover, modeling the tip antenna response as a simple electronic circuit, we prove that these electrical characteristics can be calculated taking into account the tip radius and the nanometric field penetration depth. Using numerical simulations we calculate the field enhancement factor as a function of the excitation frequency and we show that it is related to the high field screening at the apex of the nanostructure. Coupling this result with the electronic circuit calculations, we introduce a method to predict the near $\mathrm{THz}$ field waveform at the nanotip surface.

The experimental setup used in this study is composed of a pulsed $\mathrm{THz}$ source and an ultrafast near infrared (NIR) laser source, which both illuminate a nanoscale Tungsten tip biased at negative DC voltage inside a vacuum chamber to trigger electrons field emission, similarly to the setup of ref.[11]. These emitted electrons are collected on a particle detector (two microchannel plates) coupled with a lock-in amplifier. The NIR laser source is a Spitfire femtosecond laser (Spectra Physics) generating sub-50 fs pulses at $800 \mathrm{~nm}$ wavelength with 
a repetition rate of $1 \mathrm{kHz}$ and an energy up to $4 \mathrm{~mJ} /$ pulse. The beam is split in two parts (90/10 in energy), the highest energy beam is used for THz generation. The low energy beam is used as probe for $\mathrm{THz}$ pulse characterization by Eelectro-optic (EO) samplig using a GaP crystal or as pump for electron emission. The THz pulse is generated thanks to the main NIR beam using the two color plasma generation process[6]. The THz beam is sent to the EO detector to be characterized or to the analysis chamber. For the field emission analysis, the $\mathrm{THz}$ beam is focalized on the nanoscale $\mathrm{W}$ biased tip with a spherical mirror. The $\mathrm{W}$ tip is prepared by standard electro-polishing method. The temporal traces of the generated $\mathrm{THz}$ pulses, characterized with the EO detection system, are reported in Fig.1(a). Measurements are conducted for two different polarities of the $\mathrm{THz}$ pulses. The pulse polarity is controlled by acting on the phase difference between the NIR wave and its second harmonic as reported in ref.[11]. During the experiment, the $\mathrm{W}$ tip is biased at $-105 \mathrm{~V}$ and both the NIR laser and $\mathrm{THz}$ beams are focalized on the tip. A delay line is used to tune the delay between the NIR and the $\mathrm{THz}$ pulses to proceed to a pump-probe experiment. Electrons are emitted and detected: the $e$-lock-in signal is of $1.12 \mathrm{mV}(0.1 \mathrm{mV})$ when only NIR pulses (only $\mathrm{THz}$ pulses) are used.

As shown in Fig.1(c), when only NIR pulses are focused on the sample, the lock-in signal related to the detection of emitted electrons increases linearly as function of the applied DC voltage, at constant laser power, as reported in literature for multi-photon photoemission[18]. Focusing also the $\mathrm{THz}$ pulses on the sample, the pump-probe measurements are performed at fixed bias of $-105 \mathrm{~V}$ and are reported in Fig.1(b): the electron current signal is recorded for both $\mathrm{THz}$ polarities. At short delays, between -5 and $-2 \mathrm{ps}$, a constant value of the signal is measured $(1 \mathrm{mV})$. It corresponds to the electronic emission without $\mathrm{THz}$ contribution, only due to the static voltage of $-105 \mathrm{~V}$ and the NIR illumination. Therefore, the $\mathrm{THz}$ pulse has no influence on the $e$-emission signal. For delays longer than $-2 \mathrm{ps}$, the emission current is affected by the $\mathrm{THz}$ pulse: its field is combined with the static field produced by the $-105 \mathrm{~V}$ at the nanoscale tip when the NIR pulse reach the sample. When the THz field is negative as the static field, they add and the field emission is enhanced. On the other way, when the THz field is positive, it is subtracted to the static field and the emission strength is reduced. We consider that the static bias and the THz electric field are independent; such an hypothesis has already been discussed in previous works $[17,19]$. If the $\mathrm{THz}$ positive field is strong enough, the emission is cancelled. This effect is clearly visible with the two opposite 
(a)

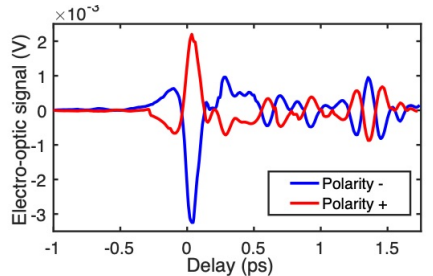

(b)

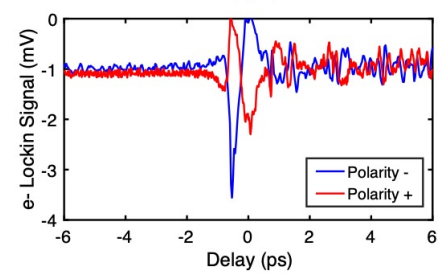

(c)

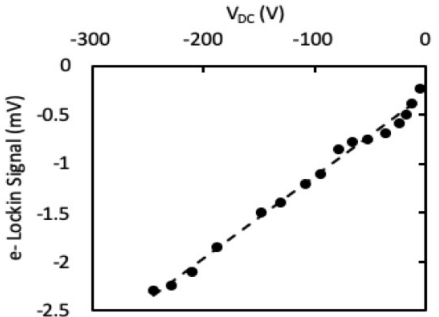

FIG. 1. (a)Electro-optic characterization of $\mathrm{THz}$ pulses measured for both polarities with a lockin amplifier connected on two balanced photodiodes; (b)Evolution of the electronic emission signal in pump probe experiment at a tip bias of $-105 \mathrm{~V}$. The NIR pump is set at $8 \mathrm{~nJ}$ and focused on $4 \mu \mathrm{m}$ spot for 50 fs pulse duration and the THz probe is the same as (a); (c) Evolution of the electron current signal as a function of the negative applied bias under $8 \mathrm{~nJ}, 50 \mathrm{fs}$ IR pulses focused on 4 $\mu \mathrm{m}$ spot.

polarities of the THz pulse in Fig.1(b). Thanks to the results of Fig1(c), a correspondence between DC voltage and emission signal can be established and used to convert the measured lock-in signal in Fig.1(b) into equivalent static bias $\left(V_{D C}^{T H z}\right)$ applied to the tip.

The near $\mathrm{THz}$ field is calculated using the relation:

$$
F_{n e a r}=\frac{V_{D C}^{T H z}}{\beta r}
$$

where $r$ is the tip radius and $\beta$ a geometrical factor. The product $\beta r$ was experimentally measured to be about $250 \mathrm{~nm}$, by performing, from the same sample, field ion emission 
measurements see ref.[20]. For the two polarities of the incident THz pulse, the near THz field temporal behavior is reported in Fig.2(a-b) together with the incident THz field, calibrated using the formula:

$$
F_{\text {in }}=\left(\frac{A-B}{A+B}\right) \frac{\lambda_{s}}{2 \pi r_{41} t_{c} L_{c} n_{s}^{3}}
$$

with $A$ and $B$ are the signal intensities of the balanced photodiodes, $\lambda_{s}=800 \mathrm{~nm}$ is the pump wavelength, $t_{c}=0.46$ the transmission factor of the GaP crystal, $L_{c}=0.1 \mathrm{~mm}$ the crystal thickness, $r_{41}=0.88 \mathrm{pm} / \mathrm{V}$ its non-linear susceptibility factor and $n_{s}=3.18$ its refraction index.

Therefore, the $\mathrm{THz}$ input signal of $1.2 \mathrm{~V} / \mu \mathrm{m}$, which endures $30 \%$ attenuation within the entry window of the vacuum chamber, is enhanced up to $1.2 \mathrm{~V} / \mathrm{nm}$, at the tip apex, corresponding to an enhancement field factor $\eta$ of about 1400. By comparing the THz near field waveform $\left(F_{\text {near }}\right)$ with the $)$ EO sampling measurement of the incident $\mathrm{THz}$ field $\left(F_{\text {in }}\right)$, it is possible to observe that the half width of the main peak is about $(120 \pm 20)$ fs for the $F_{\text {in }}$ signal and $(260 \pm 20)$ fs for the $F_{\text {near }}$ signal (see Fig.2(a)).

The corresponding spectra of the EO sampling and the $e$-emission signals, calculated by FFT, are reported in Fig.3. The spectrum of EO signal $\left(F_{i n}\right)$ strongly decreases for frequencies larger than $8 \mathrm{THz}$, due to the GaP optical phonon absorption. The spectrum of the $e$-emission signal $\left(F_{\text {near }}\right)$ is shifted to lower frequencies values between 0.1 and $2 \mathrm{THz}$ and its amplitude decreases by a factor 10 for frequencies higher than $4 \mathrm{THz}$.

As discussed in previous scanning near-field optical microscopy[14, 16] and THz streaking spectroscopy investigations of a nanotip[11], the THz near field is phenomenologically simulated using a generic antenna-model $[11,14,16]$, where an equivalent RLC circuit was assigned to the nanotip. The relationship between the $\mathrm{THz}$ near field at the tip apex $F_{\text {near }}(t)$ and the current $I_{T H z}(t)$ circulating in an antenna is given as follows:

$$
F_{\text {near }}(t) \propto \int I_{T H z}\left(t^{\prime}\right) d t^{\prime}
$$

Similarly, the induced current $I_{T H z}(\omega)$ in the frequency domain is expressed as:

$$
I_{T H z}(\omega) \propto \frac{F_{i n}(\omega)}{R+j \omega L-j /(\omega C)},
$$

where $F_{i n}(\omega), R, L$ and $C$ are the incident THz far field, radiation resistance, inductance and capacitance, respectively. The resonance frequency is determined from $f_{0}=1 /(2 \pi \sqrt{L C})$. 

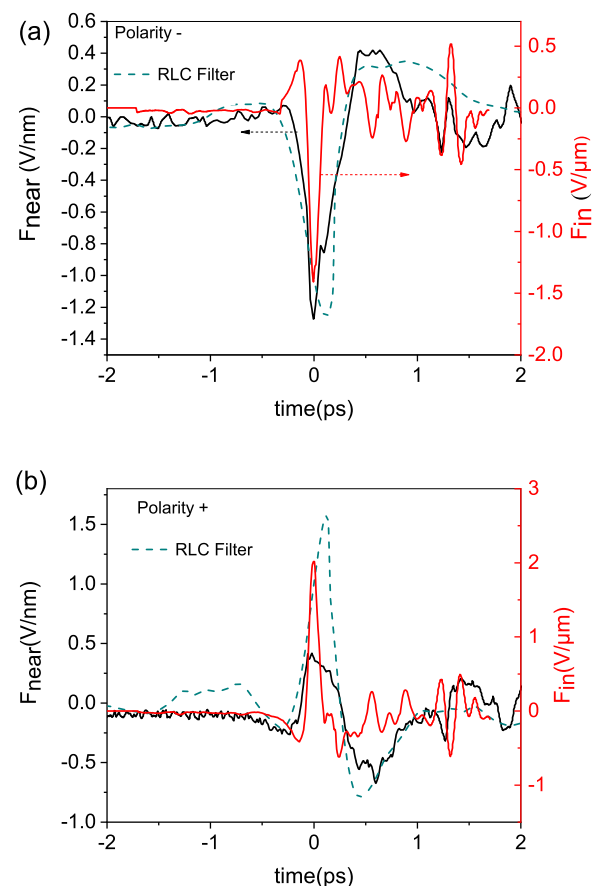

FIG. 2. The THz near field waveform (black), the incident $\mathrm{THz}$ field waveform $F_{\text {in }}$ (red) and the reconstructed waveform using RCL filter with $R=144 \Omega, C=0.7 \mathrm{fF}, L=0.04 \mathrm{nH}$ (green), for negative polarity (a) and positive polarity (b).

Because we measure the incident $\mathrm{THz}$ field outside the vacuum chamber, we cannot compare the phase shift between the $F_{\text {near }}$ and $F_{\text {in }}$, therefore we concentrate on the amplitude:

$$
F_{\text {near }}(\omega) \propto \frac{F_{\text {in }}(\omega)}{\sqrt{\left(1-x^{2}\right)^{2}+\left(\frac{x}{Q}\right)^{2}}}
$$

where $x=f / f_{0}$ and $Q=1 /(R) \sqrt{\frac{L}{C}}$. Fitting the experimental spectra of Fig.3 using Eq.3, we obtained $f_{0}=0.9 \mathrm{THz}$ and $Q=1.8$. The inductance and capacitance of a hemispherical capped cylindrical rod can be expressed as function of the radius $r$ of about $50 \mathrm{~nm}$ and the effective length $l$, as follows[12, 21, 22]:

$$
\begin{aligned}
C & =\frac{2 \pi \epsilon l}{\ln \frac{l}{2 r}+1} \\
L & =2 l\left(\log \frac{2 l}{r}-\frac{3}{4}\right) \times 10^{-7}(H)
\end{aligned}
$$



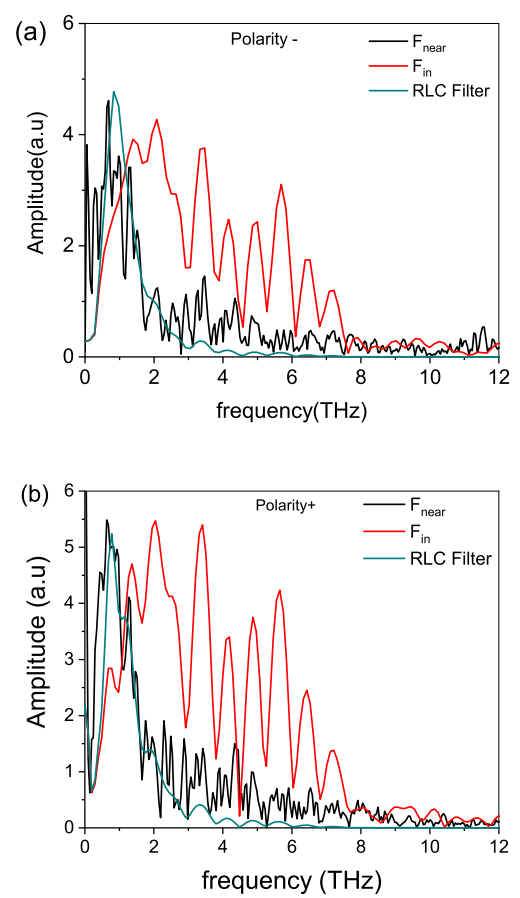

FIG. 3. Fourier transform of the $\mathrm{THz}$ near field (black) and the incident $\mathrm{THz}$ field $F_{\text {in }}$ (red) and calculated $\mathrm{THz}$ field after RLC filtering of $F_{\text {in }}$ spectrum (green), for negative polarity (a) and positive polarity (b).

The resistance is equal to that of a truncated conical of effective length $l$, half-cone angle $\theta$ and a conductive shell with thickness $\delta$ :

$$
R=\int_{0}^{l} \frac{\rho d z}{\pi\left(2 \delta(r+z \tan (\theta))-\delta^{2}\right)}=\frac{\rho}{2 \pi \delta \tan (\theta)} \log \left(\frac{2 l \tan (\theta)}{2 r-\delta}+1\right) .
$$

Replacing the Eq.4 and 5 into the expression of the resonance frequency $f_{0}$ and taking into account its experimental value, we determine the value of the effective antenna length of $l=$ $0.5 \lambda_{T H z}$, where $\lambda_{T H z}$ is the wavelength associated to $2.2 \mathrm{THz}$ corresponding to the maximum amplitude of the input spectrum of $F_{\text {in }}$ in Fig.3(a). This result is in agreement with the resonant behavior of the linear antenna observeed at $l=\lambda_{T H z} / 2, \lambda_{T H z}, 3 / 2 \lambda_{T H z}, . .[23]$. For larger cone angle nano-tip shorter effective length have been reported by Li et al.[12], however we focus here our attention of nanowire-like geometry. Using this value for the effective 
length $l$, from Eq.4 and Eq. 5 we calculate $\mathrm{C}=0.7 \mathrm{fF}$ and $\mathrm{L}=0.04 \mathrm{nH}$, respectively. Replacing the Eqs.4 to 6 in the expression of the quality factor $Q$ and using the value of $l=0.5 \lambda_{T H z}$, from the experimental value of $Q=1.8$, we calculate the thickness of the conducting shell $\delta=7 \mathrm{~nm}$ and from Eq.6 the value of the resistance $\mathrm{R}=144 \Omega$. The conduction shell thickness is really small compared to the skin depth of tungsten at $2.2 \mathrm{THz}$, which is about $80 \mathrm{~nm}$. As already reported by Seo et al.[24] the THz field almost not penetrate inside the metal nano structures, due to high surface density of charges in the nanoscale structure.

In order to confirm this behavior for nanotips and calculate the field enhancement at the apex as function of the $\mathrm{THz}$ frequencies, we numerically solve the Maxwell equations using Finite Difference Time Domain (FDTD) method on a nanometric metallic tip with an apex radius of 50 or $25 \mathrm{~nm}$, a length of 60 or $220 \mu \mathrm{m}$ and a shank angle of $2^{\circ}$. Our FDTD field simulations are performed with Lumerical commercial software[25]. The tip is illuminated with a radiation whose frequency varies from 0.5 to $256 \mathrm{THz}$. The incident electric field is fixed at $1 \mathrm{~V} / \mathrm{m}$ and the enhancement is measured at the apex surface. Concerning the refractive index of the metallic tip, we use the values reported by Palik[26] for Iron and the Perfect Conductor (PEC) parameters. The simulated electric field distribution represented in Fig.4(a-b) clearly shows that the THz field is screened at the surface on a depth of about $5 \mathrm{~nm}$ (the pixel size). This numerical results is in good agreement with the thickness value of the conduction shell of $7 \mathrm{~nm}$. Concerning the simulation of the field enhancement factor no significant difference is observed between PEC and Iron samples. Therefore, the PEC properties can be used in the following to qualitatively simulate the $\mathrm{W}$ tip. The data of Fig.4(c) show the global $1 / f$ trend. This dependence, which is universal in any capacitor problem with an alternating current source, is therefore consistent with the terahertz light field-induced, harmonically oscillating currents charging the nanotip. Therefore, the field enhancement factor follows the equation $\eta=2800 / f$. For an excitation frequency centered around $2.2 \mathrm{THz}$ (such as for our incident $\mathrm{THz}$ pulse), the field enhancement factor of 1300 is calculated, in good agreement with the value (1400) obtained experimentally at a bias of $-105 \mathrm{~V}$, indicating that the static bias does not significantly affect the results. Actually, the bias acts on the electrons distribution only at the apex, on a depth of a few nanometers[27] however the enhancement is linked to the collective response of electrons to the e.m. excitation on a spatial scale of at least the $\mathrm{THz}$ wavelength

As a conclusion, we measure the near $\mathrm{THz}$ field using an experimental method introduced 

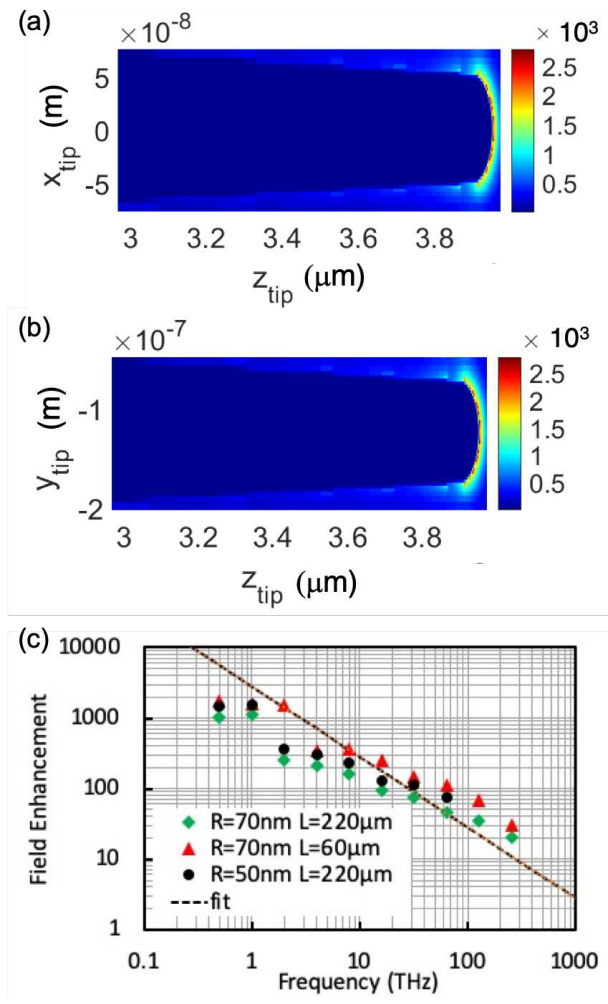

FIG. 4. Simulated electric field in the (x,z) plane (a) and (y,z) plane (b), for linearly polarized monochromatic THz pulses along the $z$-axis at $1 \mathrm{THz}$. (c) Electric field enhancement as function of $\mathrm{THz}$ frequency for different tip radiuses and lenghts. Dash line correspond to a fit with the Eq.2 with $\alpha=2800 \mathrm{THz}$ and Const $=0$.

by Yoshida et al.[17] for THz-STM, which we adapt for use in the context of multiphoton field emission. Thanks to this experimental set-up, we measure the field enhancement factor and the RCL response of the field emitter. We show that the response of the nanotip antenna can be calculated considering the cylindrical nanorod geometry for capacitance and inductance values. Concerning the resistance, we prove that the oscillating currents charging the nanotip flows on a surface layer whose thickness is thinner than the skin depth due to the high carrier concentration in the nanoscale dimensions of the tip. These results show the possibility to calculate the antenna response of the field emitter and thus to predict the 
near THz field waveform.

The experimental measurements were repeated on an aluminum field emitter, confirming that the effective length is equal to half of the excitation wavelength and showing a higher field enhancement associated to a smaller thickness of the conduction layer.

This work was supported by the French "l'Agence Nationale de la Recherche (ANR)", through the program "Investissements d'Avenir" (ANR-10-LABX-09-01), LabEx EMC3, by the European Union with the European Regional Development Fund (ERDF) and the Regional Council of Normandie and by Carnot Institut ESP

The data that support the findings of this study are available from the corresponding author upon reasonable request

* angela.vella@univ-rouen.fr

[1] P. Salén, M. Basini, S. Bonetti, J. Hebling, M. Krasilnikov, A. Y. Nikitin, G. Shamuilov, Z. Tibai, V. Zhaunerchyk, and V. Goryashko, Physics reports 836, 1 (2019).

[2] B. Salem, D. Morris, V. Aimez, J. Beerens, J. Beauvais, and D. Houde, Journal of Physics: Condensed Matter 17, 7327 (2005).

[3] M. Tani, S. Matsuura, K. Sakai, and S.-i. Nakashima, Applied optics 36, 7853 (1997).

[4] J. Fülöp, L. Pálfalvi, G. Almási, and J. Hebling, Optics express 18, 12311 (2010).

[5] C. Vicario, B. Monoszlai, and C. P. Hauri, Physical review letters 112, 213901 (2014).

[6] T. Bartel, P. Gaal, K. Reimann, M. Woerner, and T. Elsaesser, Optics Letters 30, 2805 (2005).

[7] I. Dey, K. Jana, V. Y. Fedorov, A. D. Koulouklidis, A. Mondal, M. Shaikh, D. Sarkar, A. D. Lad, S. Tzortzakis, A. Couairon, et al., Nature communications 8, 1 (2017).

[8] M. Liu, H. Y. Hwang, H. Tao, A. C. Strikwerda, K. Fan, G. R. Keiser, A. J. Sternbach, K. G. West, S. Kittiwatanakul, J. Lu, et al., Nature 487, 345 (2012).

[9] K. Iwaszczuk, M. Zalkovskij, A. C. Strikwerda, and P. U. Jepsen, Optica 2, 116 (2015).

[10] S. Mastel, M. B. Lundeberg, P. Alonso-González, Y. Gao, K. Watanabe, T. Taniguchi, J. Hone, F. H. Koppens, A. Y. Nikitin, and R. Hillenbrand, Nano letters 17, 6526 (2017).

[11] L. Wimmer, G. Herink, D. R. Solli, S. V. Yalunin, K. Echternkamp, and C. Ropers, Nature Physics 10, 432 (2014). 
[12] S. Li and R. Jones, Nature communications 7, 1 (2016).

[13] G. Herink, L. Wimmer, and C. Ropers, New Journal of Physics 16, 123005 (2014).

[14] K. Wang, D. M. Mittleman, N. C. van der Valk, and P. C. Planken, Applied physics letters 85, 2715 (2004).

[15] K. Yoshioka, I. Katayama, Y. Arashida, A. Ban, Y. Kawada, K. Konishi, H. Takahashi, and J. Takeda, Nano letters 18, 5198 (2018).

[16] H.-T. Chen, S. Kraatz, G. Cho, and R. Kersting, Physical review letters 93, 267401 (2004).

[17] S. Yoshida, H. Hirori, T. Tachizaki, K. Yoshioka, Y. Arashida, Z.-H. Wang, Y. Sanari, O. Takeuchi, Y. Kanemitsu, and H. Shigekawa, ACS Photonics 6, 1356 (2019).

[18] L. A. DuBridge, Physical Review 43, 727 (1933).

[19] L. Wimmer, O. Karnbach, G. Herink, and C. Ropers, Physical Review B 95, 165416 (2017).

[20] F. Vurpillot, in Atom Probe Tomography, edited by W. Lefebvre-Ulrikson, F. Vurpillot, and X. Sauvage (Academic Press, 2016) pp. $17-72$.

[21] T. Sandu, G. Boldeiu, and V. Moagar-Poladian, Journal of Applied Physics 114, 224904 (2013).

[22] F. W. Grover, Inductance calculations: working formulas and tables, Vol. 974 (Isa, 1981).

[23] B. Hecht, P. Muehlschlegel, J. Farahani, H.-J. Eisler, and D. Pohl, in Tip Enhancement (Elsevier, 2007) pp. 275-307.

[24] M. A. Seo, Nature Photonics, 3, 152 (2009).

[25] L. Solutions, Computer software. Vers 8 (2017).

[26] D. P. Edward and I. Palik, "Handbook of optical constants of solids," (1985).

[27] E. Silaeva, L. Arnoldi, M. Karahka, B. Deconihout, A. Menand, H. Kreuzer, and A. Vella, Nano letters 14, 6066 (2014). 
(a)

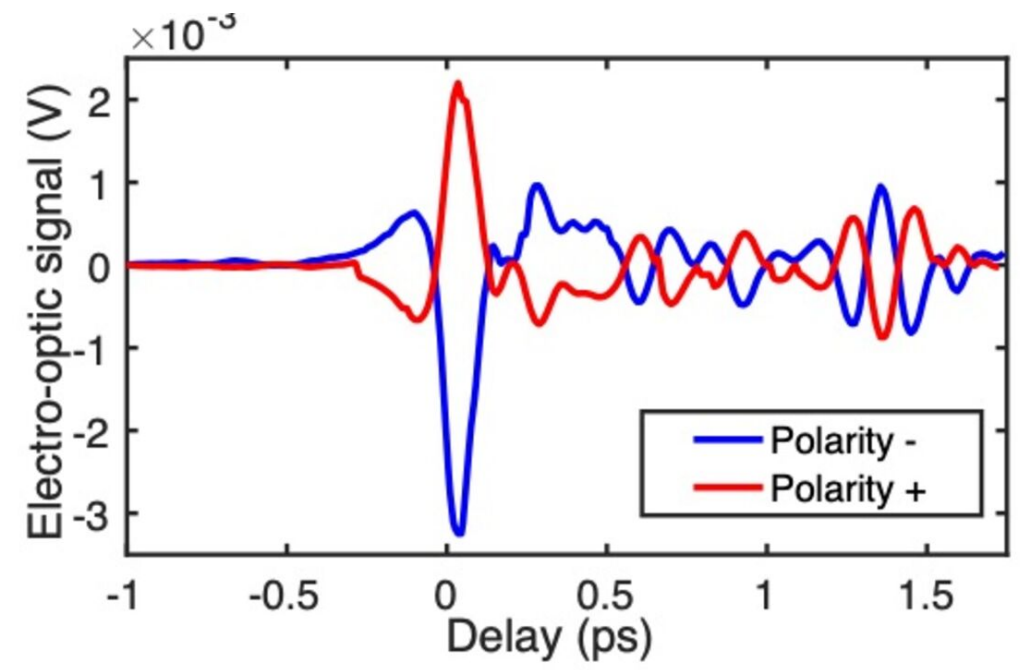

(b)

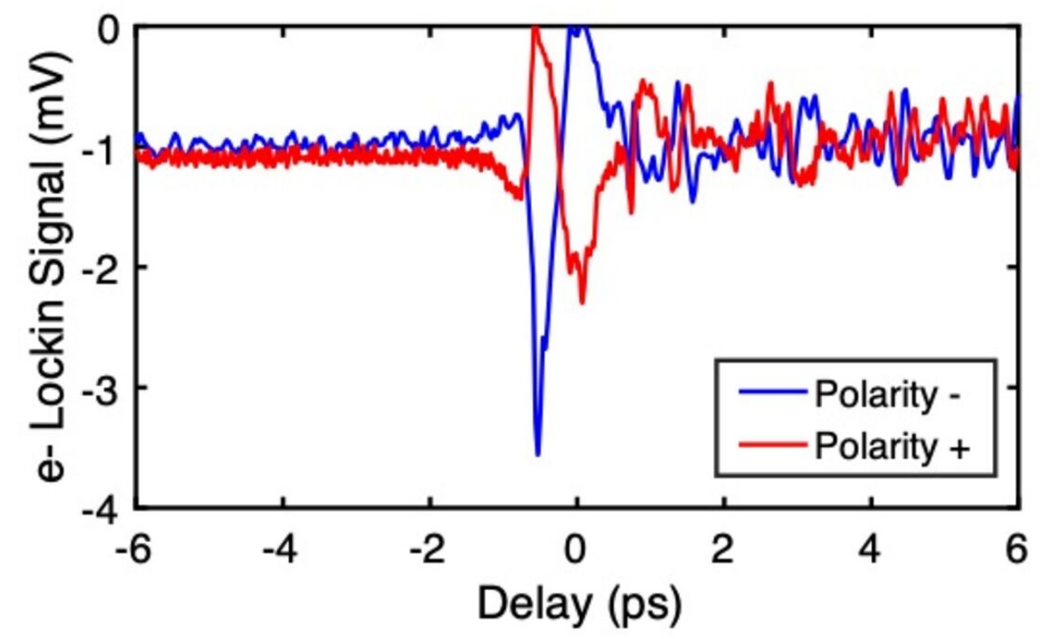

(c)

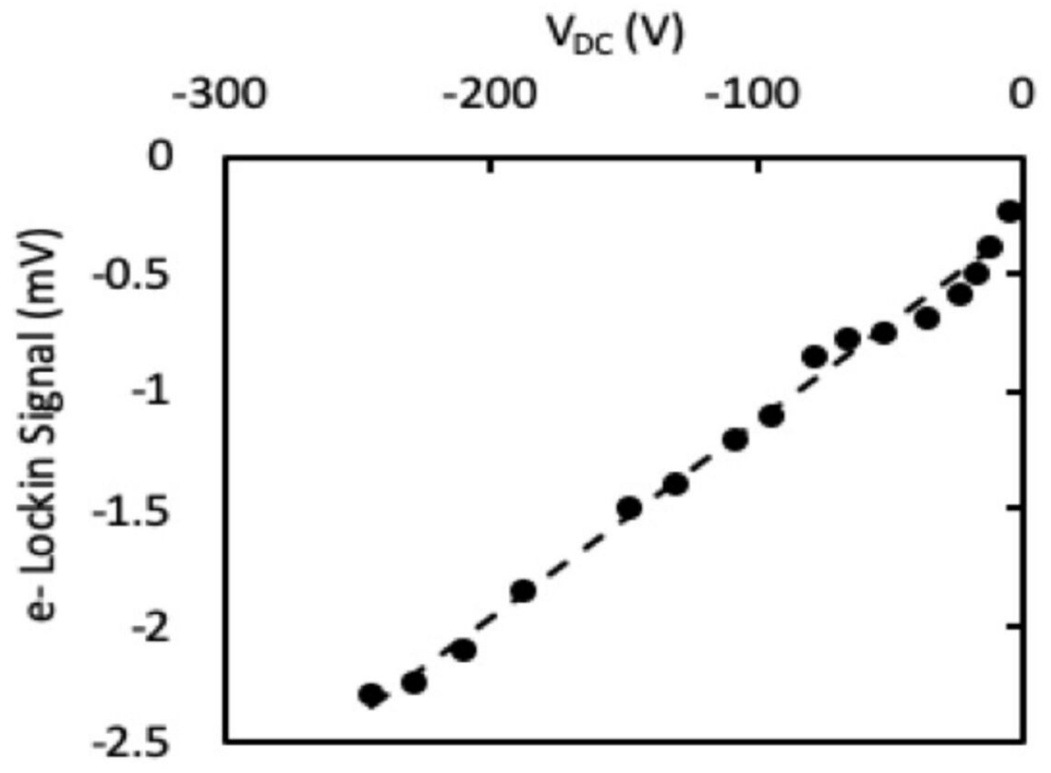




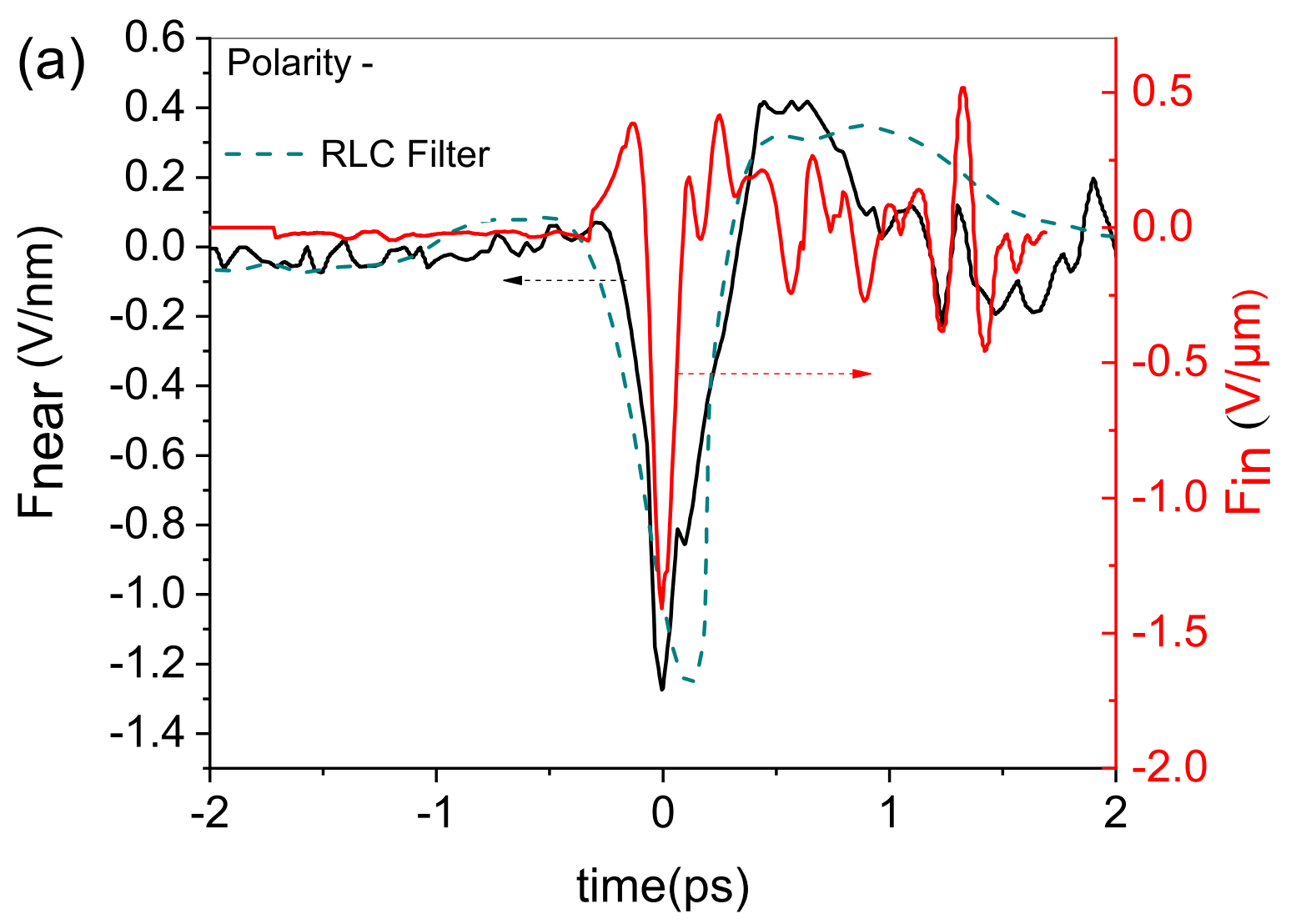




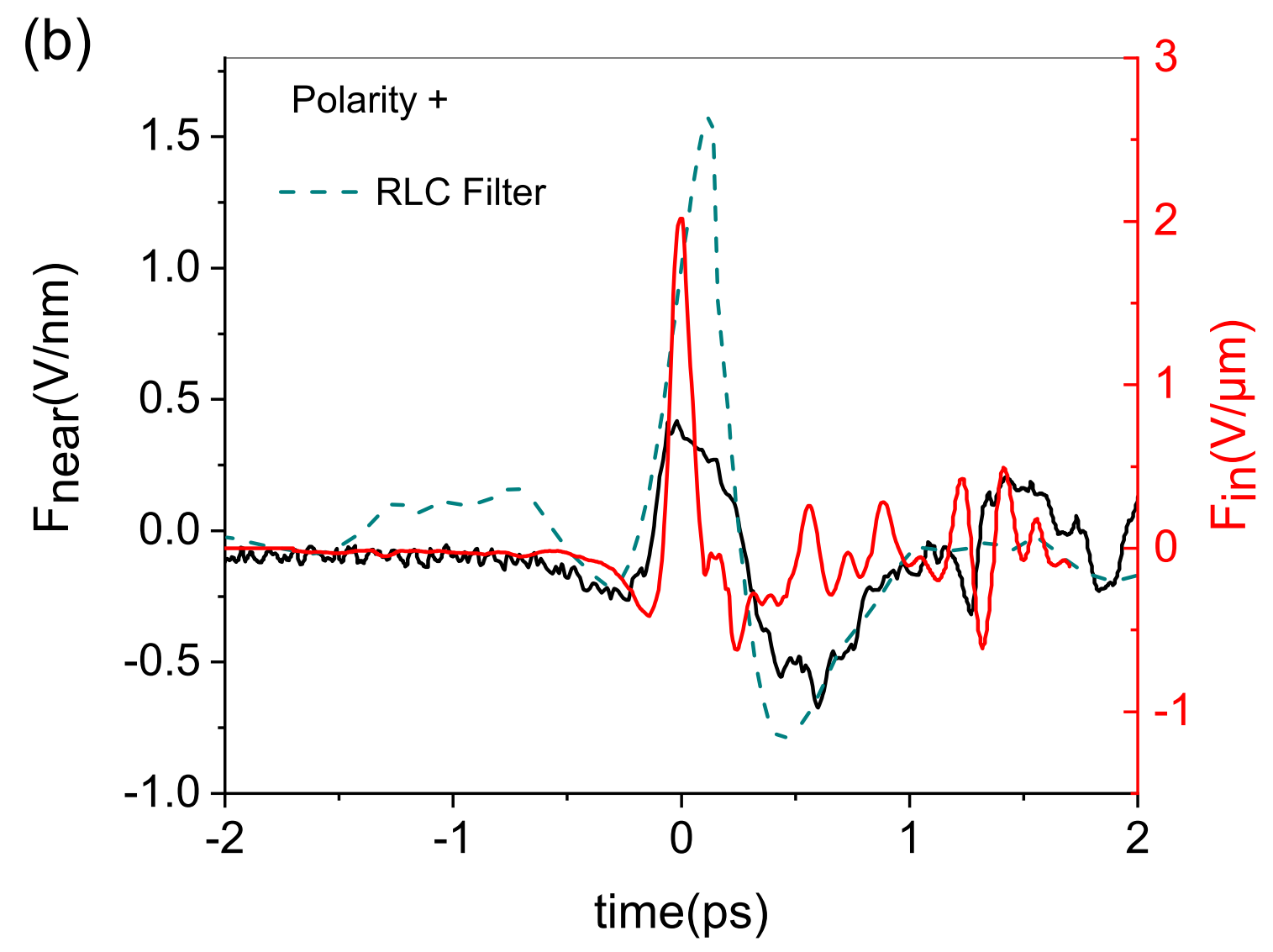

兄喜产 


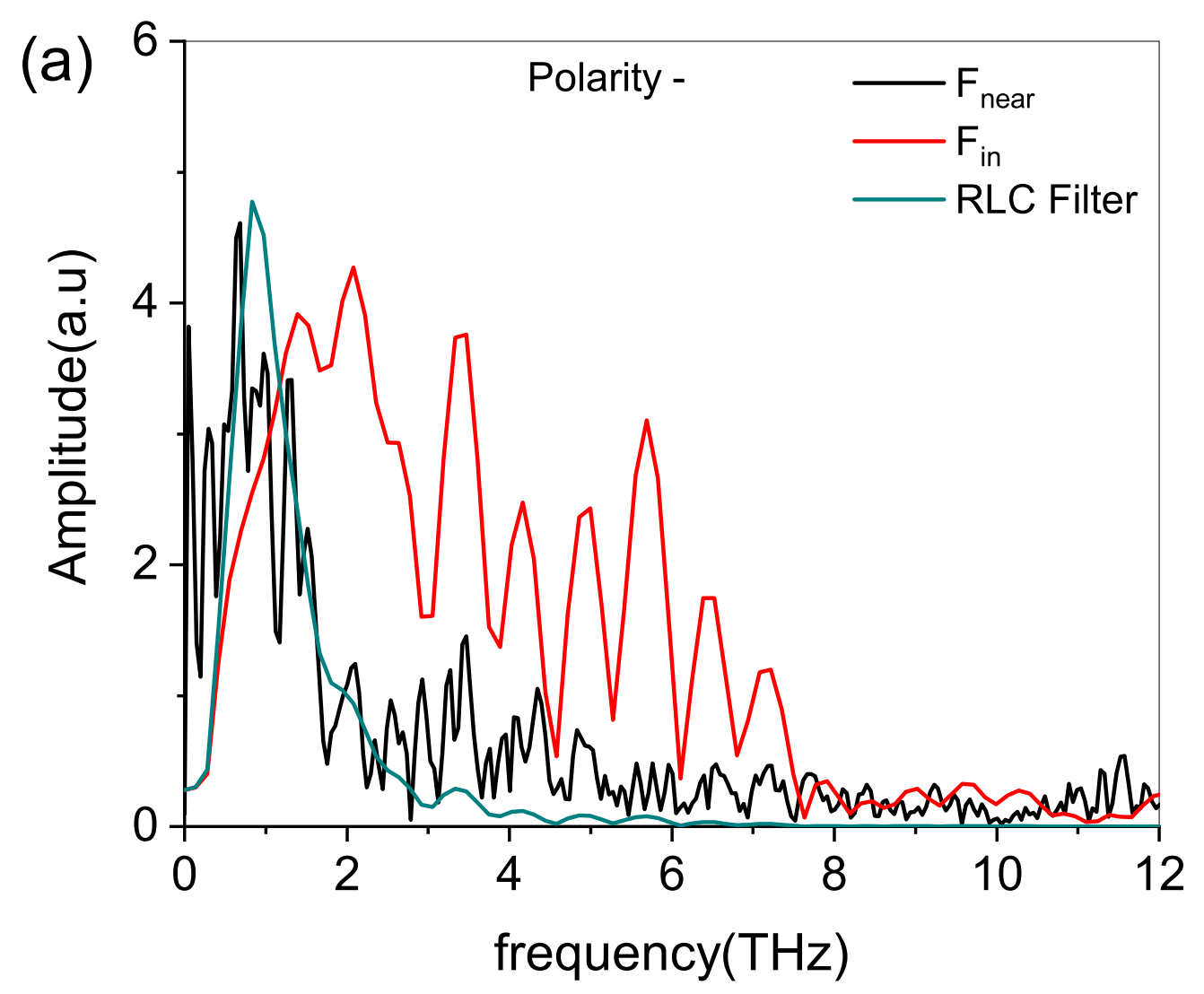

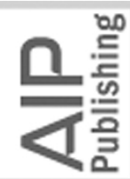




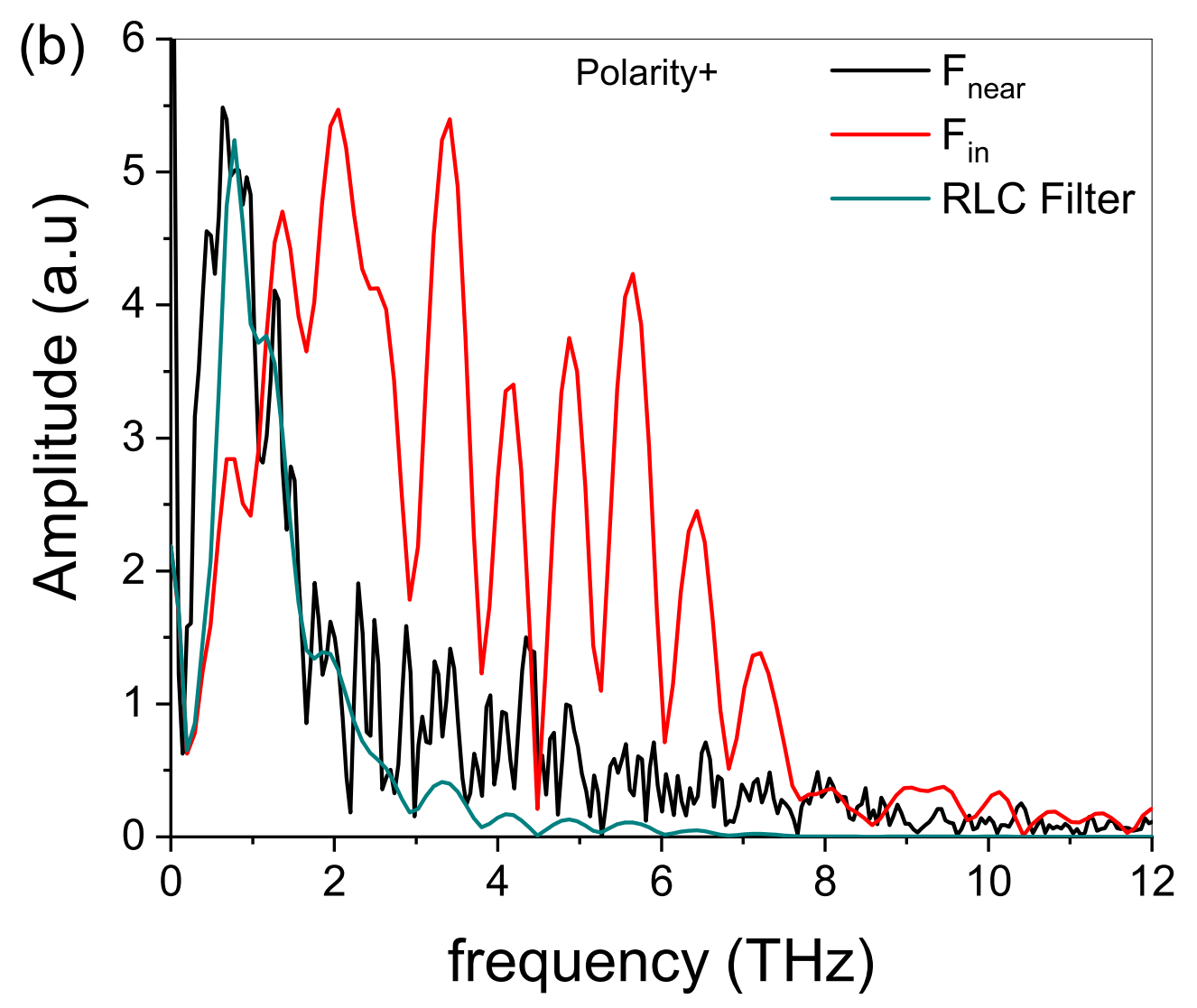

는 
(a)

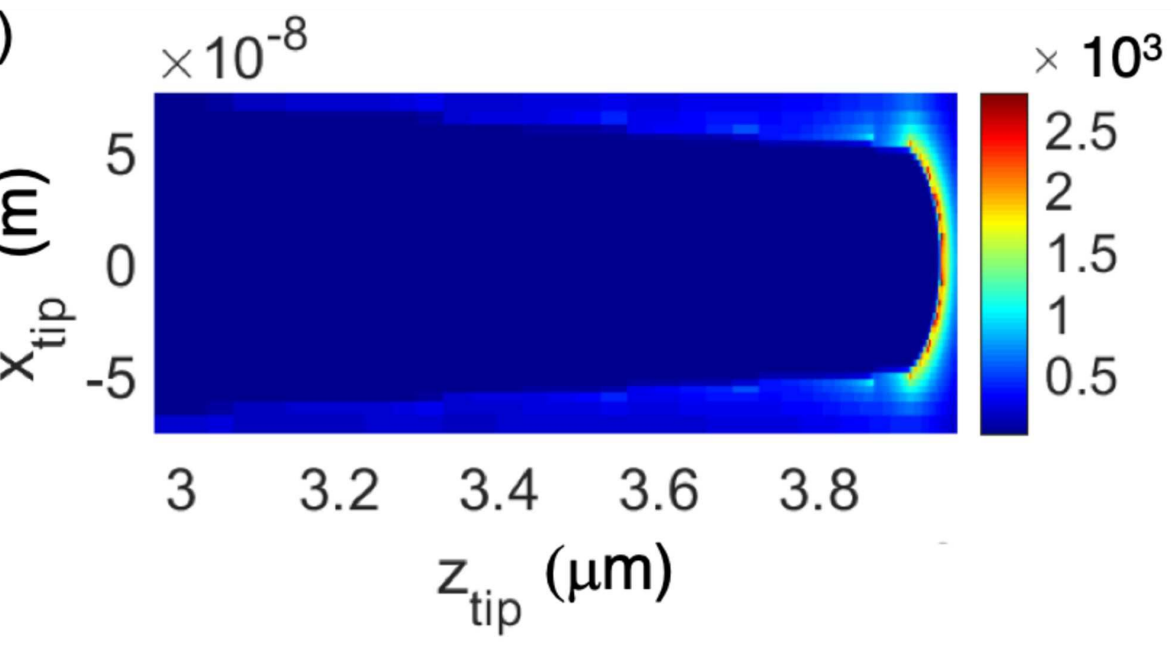

(b)

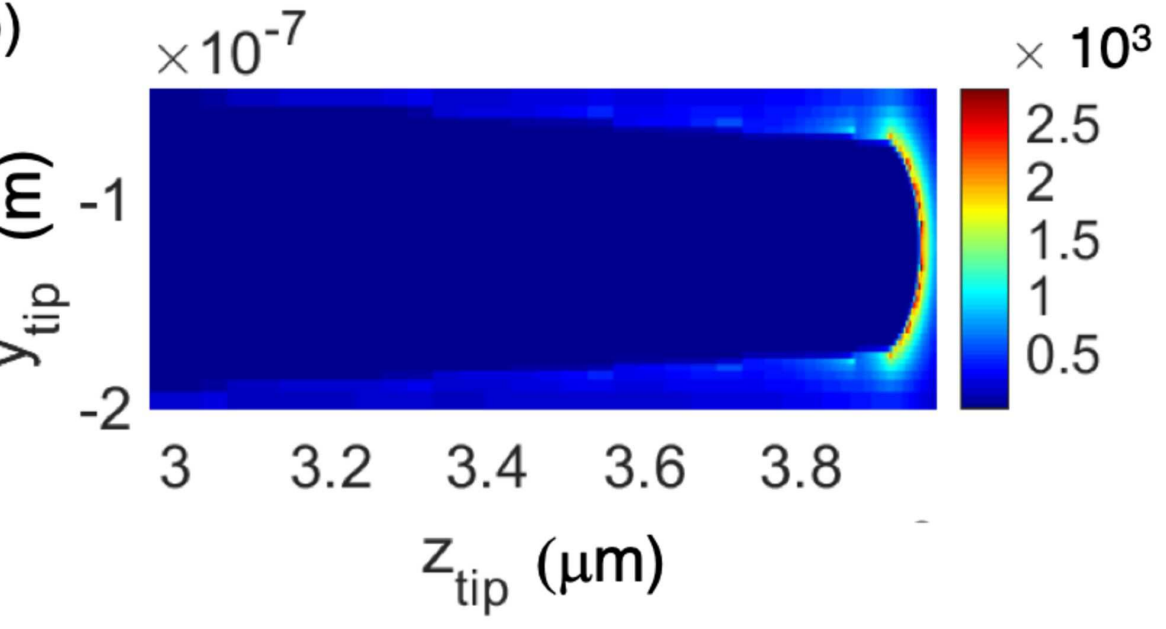

(c) 10000

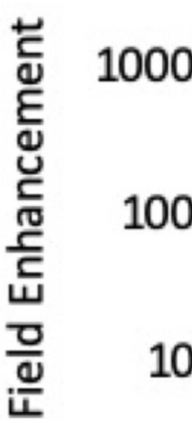

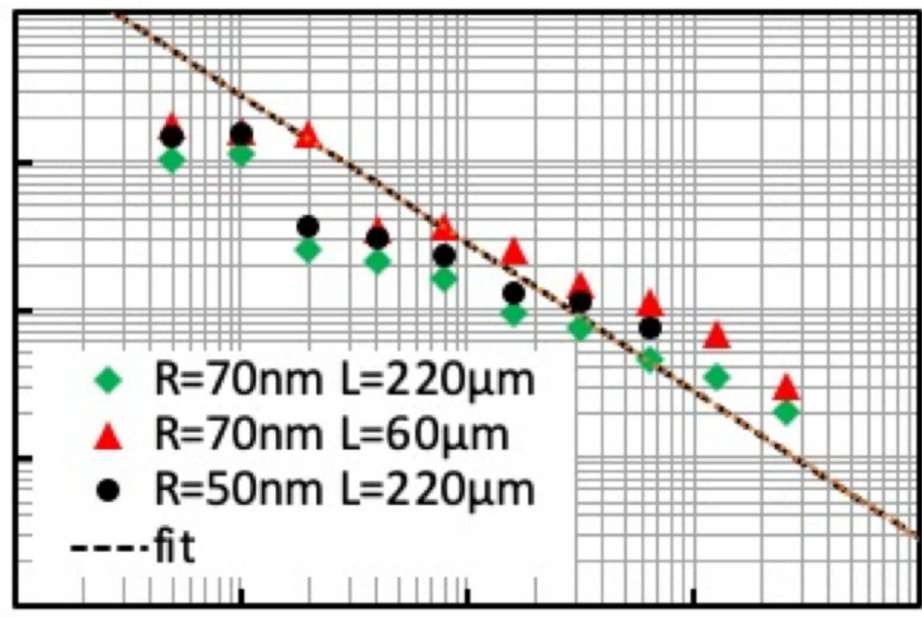

0.1

$1 \underset{\text { Frequency }(\mathrm{THz})}{100}$ 